\title{
The statistics of Sco X-1 kHZ QPOs
}

\author{
TOMASZ BULIK ET AL. \\ Nicolaus Copernicus Astronomical Center, Bartycka 18, 00716 Warszawa, Poland
}

Received; accepted; published online

\begin{abstract}
Recently an additional technique was applied to investigate the properties of $\mathrm{kHz}$ QPOS, i.e. the analysis of the distribution of frequency ratios or frequencies themselves. I review the results of such work on the data from Sco X-1: Abramowicz et al. (2003), which was later criticized by(Belloni et al., 2005). I find that the findings of the latter paper are consistent with results presented earlier: $\mathrm{kHz}$ QPOs cluster around the value corresponding to the frequency ratio of $2 / 3$. I also discuss the random walk model of $\mathrm{kHz}$ QPOs and possible future observations needed to verify it.
\end{abstract}

Key words:

(c) 0000 WILEY-VCH Verlag GmbH \& Co. KGaA, Weinheim

\section{Introduction}

The nature of $\mathrm{kHz}$ quasi periodic oscillations continues to puzzle astrophysicists. In order to unveil the nature of these phenomena different quatities are considered. For example the difference of the two $\mathrm{kHz}$ QPO, which in the beat frequency model is constant was analyzed. The disk resonance model e.g. Abramowicz and Kluźniak (2001) predicts that the ratio of the two $\mathrm{kHz}$ QPO frequencies should assume a set of discete values. In order to test it we analyzed the statistical properties of KHz QPOs in Sco X-1 (Abramowicz et al., 2003). We have found that the frequency ratio of the lower to higher QPO cluster around 0.688 , a value close to the canonical 2/3 seen in the QPOs from black hole candidates. This discovery has recently been disputed by Belloni et al. (2005).

In this paper I will discuss the similarities and differences of the two analyses. In section 2 I present an overview of different methods used to compare distributions and in section 3 I summarize the results of Abramowicz et al. (2003). I compare our results with the work of (Belloni et al., 2005) in section 4. Section 5 contains summary and a discussion of the possible future observational tests.

\section{Comparison of distributions}

Inferring the shape of an underlying distribution given a set of observed quantities is a diffcult task. In a number of cases we have no prior knowledge of what the shape of the distribution

Correspondence to: bulik@camk.edu.pl should be. Very often we do not know if the measured quantity is fundamental from the physical point of view. However, one can start by making simple assumptions about the data and testing them.

There is a number of ways to compare the shape of two distributions e.g. a model and an observed one. The $c h i^{2}$ test comparing a binned observed distribution with a model are not very useful. Such comparison suffer from a number of arbitrary assumptions about the widths of the bins which makes the results not reliable. A different set of statistical tests that do not require binning of the data is based on comparing cumulative distributions. One can introduce various metrics to measures distance between two cumulative distributions and calculate the probability distribution of such distance. A very useful test using this approach is the Kolmogorov-Smirnov test in which the metric is defined as

$D_{K S}=\max \left|\left(C_{1}-C_{2}\right)\right|$,

where $C_{i}$ are the cumulative distributions. The sensitivity of such test is limited, yet it serves as a convenient tool for comparing distributions and assessing goodness of fit. There exist modified and more sensitive versions of this test. For example the Fiszer-von Mises tests uses the metric defines as

$D_{\text {Fisz }}=\int\left(C_{1}-C_{2}\right)^{2}$,

and is therefore much more sensitive to differences in overall shape of two distributions. However calculating the probabilty distribution of $D_{\text {Fisz }}$ is a much more demanding task/

Any such test can be used for parameter estimation. Suppose that the model distribution $C_{m}\left(p_{k}\right)$ is a function of several parameters $p_{k}$. We can then maximize the probability of 

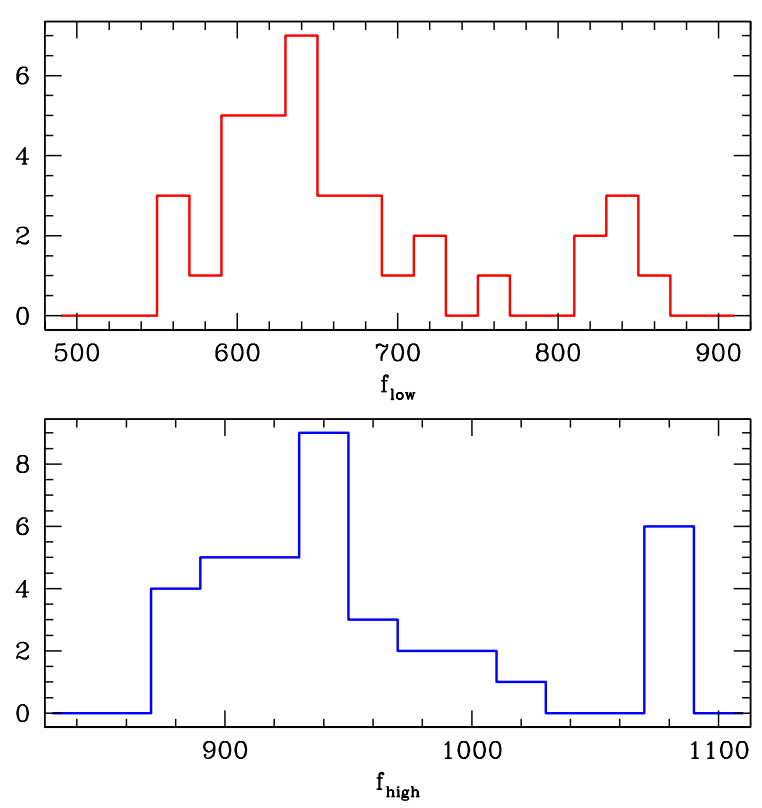

Fig. 1. The binned distributions of the lower and upper QPO frequencies from the data set ofvan der Klis et al. (1997). Note that the two distributions are similar but not identical.

the model given the observational set of data. In this case however the most sensitive method to use is the maximum likelihood. In this approach we can calculate the probability of the model $M$ given the data $X:\left(x_{l}\right)$ :

$\mathcal{L}(M \mid X) \propto \Pi \frac{d p}{d x}\left(x_{l}\right)$.

This likelihood function can be maximized with respect to the model parameters to yield the best fit model. The goodness of fit of such model can evaluated using e.g. the Kolmogorov Smirnov test. In order to find the probability distribution of the parameters one ususally assumes that it is proportional to the likelihood function.

\section{Summary of the Sco X-1 analysis}

In our paper we have addressed the question whether there is any evidence of clustering of the frequency ratios of $\mathrm{kHz}$ QPOs in Sco X-1. To do that we used a set of data published by van der Klis et al. (1997). This set contained 39 frequency pairs and was used by the authors to prove that the difference between the two frequencies is not constant. We have first verified the hypothesis that the distribution of frequency ratios is uniform using the Kolmogorov-Smirnov test. This probability is

$P_{\text {uniform }}=0.00047$,

which led us to consider the question if the frequency ratios cluster. We have no a priori knowledge of the shape of the distribution we are looking for as there is no physical model that we could refer to. Therefore we decided to model the shape of the distribution with a Lorentzian profile normalized on the interval $(0,1)$. We have used the maximum likelihood method to find the best fit which was a Lorentzian centered

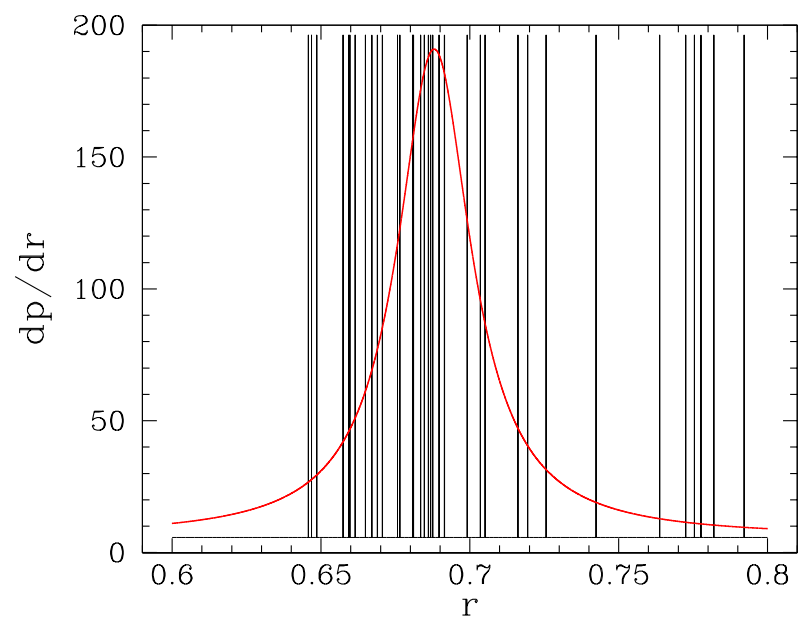

Fig. 2. The frequency ratios used by Abramowicz et al. (2003) are represented by the vertical lines. The solid line corresponds to the best fit single Lorentzian.

on $r_{0}=0.688$ with a width of $\lambda=0.0151$. The KolmogorovSmirnov test provided a confirmation that the data could have been drawn from such distribution, since the probability was

$P_{\text {Lorentzian }}=0.678$

We have also shown that there possibly is another peak in the data at $r \approx 0.78$, yet is was not significant.

\section{Comparison with the later study}

In a recent paper Belloni et al. (2005) criticize the results obtained above. There is a relation between the two $\mathrm{kHz}$ QPO frequencies, and therefore it is equivalent to analyze the distribution of one or of another frequency. The relation between the two $\mathrm{kHz}$ QPO frequencies is clearly visibly and could already be noted in van der Klis et al. (1997). The statement would have been true if the relation was exact, however this is just a correlation and not a strict one to one correspondence. One can verify that by checking the consistency of the distributions of the lower and upper frequency with the hypothesis that they are distributed uniformly, in an interval between the lowest the highest frequency in a given set. For the upper frequency we obtain the same result as Belloni et al. (2005), i.e. $P_{\text {upper }}=0.017$, while the analysis of the distribution of the lower frequency gives $P_{\text {lower }}=0.0012$. In their conclusions Belloni et al. (2005) write that the "distribution of QPO frequencies used by Abramowicz etal 2003 differs from constant can only be rejected at $2.4 \sigma$ level" This statement is only true for the distribution of the upper frequency and not for the lower. Moreover we never considered the distributions of individual frequencies in Abramowicz et al. (2003) but their ratio. The distribution of the frequency ratios differs from uniform at a level of $3.5 \sigma$. Therefore the statement that "the results of Abramowicz et al. 2003 are not statistically founded" is false.

Belloni et al. (2005) analyze the distribution of the upper kHz QPO in Sco X-1 using a larger data set. They found 
that the distribution of this QPO is not compatible with a uniform level and the fit the distribution using three gaussians. It is interesting to note that in all cases there is a peak in the distributions they obtain corresponding to the $3 / 2$ ratio of the frequencies assuming the linear relation between the two frequencies. The distribution of the frequency ratios can be transformed to the distribution of an individual frequency using the lines relation. Using the propagation of errors we estimated that there should be a peak in the distribution of upper QPO in Sco X-1 at the frequency

$\nu_{\text {peak }}=940 \pm 25 \mathrm{~Hz}$.

Belloni et al. (2005) found a peak at $432.5 \pm 1.5 \mathrm{~Hz}$ in complete agreement with our results. It is extremely interesting to find that the distributions of QPO frequencies in other sources analyzed by Belloni et al. (2005) also show clustering and peaks at the location consistent with the value of $3 / 2$, when the linear relation between the two $\mathrm{kHz}$ QPOs is taken into account.

Belloni et al. (2005) propose an explanation for the clustering of frequencies as a simple randon walk. In their model they start from $700 \mathrm{~Hz}$ and let the frequency wander in frequency in steps of $\pm 6 \mathrm{~Hz}$. They show that this model qualitatively explains clustering of frequencies. This model is not defined in detail, as it has not been clearly stated for how many steps the frequency is allowed to wander, or if there are any boundaries outside of which the frequency can not drift. Moreover in a real observation the starting point depends on a particular moment of the observation. The distributions of the upper kHz QPO of Belloni et al. (2005) is similar to the of van der Klis et al. (1997), and the Kolmogorov-Smirnov test shows that the probability that they come from the same underlying distribution is $22 \%$. One can ask a question how probable is it to obtain two such similar distributions in two realizations of a random walk. We have preformed a simulation and found that the probability of obtaining two such similar distributions varies between $10^{-4}$ and $10^{-2}$ depending on the specific prescription of the random walk.

\section{Summary and future observations}

We have shown that the analysis of the distribution of the upper kHz QPO frequencies in Sco X-1 by Belloni et al. (2005) leads to the results in total agreement with the results of Abramowicz et al. (2003). The peaks in the distribution of upper kHz QPO frequency found by Belloni et al. (2005) correspond exactly to the estimates based the earlier results of Abramowicz et al. (2003). The deviation from a uniform distribution of the frequency ratios is at the $3.5 \sigma$ level, and not $2.4 \sigma$, which corresponds to the distribution of the upper $\mathrm{kHZ}$ QPO as claimed by Belloni et al. (2005). the distribution of the lower kHZ QPO differs from uniform at a $3.2 \sigma$ level. The difference comes from the fact that the linear relation between the two frequencies is not exact but has some spread.

The random walk model proposed by Belloni et al. (2005) reproduces qualitatively the distribution of upper $\mathrm{KHz}$ QPO. The problem that it faces comes from the fact that it is very difficult to reproduce a similar distribution in two separate observations, while the set of data in van der Klis et al.
(1997) is consistent with the set of Belloni et al. (2005). This model can be verified with a long term monitoring of sources with $\mathrm{kHz}$ QPOs and a uniform sample of their behavior. If the frequencies, and frequency ratios really do cluster on some values such behavior should be reproducible in consecutive long observations. In the random walk model the peaks in the distributions of frequency ratios will wander from one observation to another. Such study must be treated with caution as we have no information on the fundamental scale of variability of $\mathrm{kHz}$ QPOs.

Furthermore in my opinion there is no preferred way of analyzing the data. There is no reason a priori to say that the frequencies or frequency ratios, their sum or difference is in any way a better quantity to analyze until we have a physical model to consider. Abramowicz et al. (2003) based the analysis on such a physical model in which the frequency ratio plays a fundamental role. The analysis by Belloni et al. (2005) is phenomenological and does not refer to any underlying physical model.

Finally there is the issue of the consistency of the data with the resonace theory (Abramowicz and Kluźniak, 2001). The theory predicts a preference for the $2 / 3$ ratio of the frequencies but is not saying anything yet about the shape of the expected distribution around this value. Such shape should probably depend on the details of the interaction in the inner disk. At this point we can only say that the value of the ratio $r=2 / 3$ lies within the width of the assumed shape of the distribution of frequency ratios in Sco X-1. This observation provides an additional hint to consider the resnance model in more detail.

Acknowledgements. The author thanks for the hospitality and support from Nordita and the KBN grant 2 P03D 00125.

\section{References}

Abramowicz, M. A., Bulik, T., Bursa, M., and Kluźniak, W.: 2003. Astronomy and Astropysics, 404, L21-L24

Abramowicz, M. A. and Kluźniak, W.: 2001. Astronomy and Astropysics, 374, L19-L20

Belloni, T., Méndez, M., and Homan, J.: 2005. Astronomy and Astropysics, 437, 209-216

van der Klis, M., Wijnands, R. A. D., Horne, K., and Chen, W.: 1997. ApJ , 481, L97+ 